\title{
A Common $q$-Analogue of Two Supercongruences
}

\author{
Victor J. W. Guo® and Wadim Zudilin®
}

Abstract. We give a $q$-congruence whose specializations $q=-1$ and $q=1$ correspond to supercongruences (B.2) and (H.2) on Van Hamme's list (in: $p$-Adic Functional Analysis (Nijmegen, 1996), Lecture Notes in Pure and Applied Mathematics, vol 192. Dekker, New York, pp 223-236, 1997):

$$
\begin{aligned}
& \sum_{k=0}^{(p-1) / 2}(-1)^{k}(4 k+1) A_{k} \equiv p(-1)^{(p-1) / 2}\left(\bmod p^{3}\right) \quad \text { and } \\
& \sum_{k=0}^{(p-1) / 2} A_{k} \equiv a(p)\left(\bmod p^{2}\right),
\end{aligned}
$$

where $p>2$ is prime,

$$
A_{k}=\prod_{j=0}^{k-1}\left(\frac{1 / 2+j}{1+j}\right)^{3}=\frac{1}{2^{6 k}}\left(\begin{array}{c}
2 k \\
k
\end{array}\right)^{3} \text { for } k=0,1,2, \ldots,
$$

and $a(p)$ is the $p$ th coefficient of the modular form $q \prod_{j=1}^{\infty}\left(1-q^{4 j}\right)^{6}$ (of weight 3$)$. We complement our result with a general common $q$-congruence for related hypergeometric sums.

Mathematics Subject Classification. 33D15, 11A07, $11 \mathrm{~B} 65$.

Keywords. Basic hypergeometric series, $q$-Dixon sum, $q$-congruence, supercongruence, creative microscoping.

Victor J. W. Guo was supported by the National Natural Science Foundation of China (grant 11771175). Wadim Zudilin was supported by JSPS Invitational Fellowships for Research in Japan (fellowship S19126). 


\section{Introduction}

The formula of Bauer [1] from 1859,

$$
\sum_{k=0}^{\infty}(-1)^{k}(4 k+1) A_{k}=\frac{2}{\pi}, \quad \text { where } A_{k}=\frac{1}{2^{6 k}}\left(\begin{array}{c}
2 k \\
k
\end{array}\right)^{3} \text { for } k=0,1,2, \ldots,
$$

is one of traditional targets for different methods of proofs of hypergeometric identities. Its special status is probably linked to the fact that it belongs to a family of series for $1 / \pi$ of Ramanujan type, after Ramanujan [21] brought to life in 1914 a long list of similar looking equalities for the constant but with a faster convergence. Identity $(1.1)$ is a particular instance of ${ }_{4} F_{3}$ hypergeometric summation (known to Ramanujan) but there are several proofs of it, including the original one [1] of Bauer, that do not require any knowledge of hypergeometric functions. One notable - computer - proof of (1.1) was given in 1994 by Ekhad and Zeilberger [2] using the Wilf-Zeilberger (WZ) method of creative telescoping.

It was observed in 1997 by Van Hamme [28] that many Ramanujan's and Ramanujan-like evaluations have nice $p$-adic analogues; for example, the congruence

$$
\sum_{k=0}^{(p-1) / 2}(-1)^{k}(4 k+1) A_{k} \equiv p(-1)^{(p-1) / 2}\left(\bmod p^{3}\right)
$$

(tagged (B.2) on Van Hamme's list) is valid for any prime $p>2$ and corresponds to the equality (1.1). The congruence (1.2) was first proved by Mortenson [19] using a ${ }_{6} F_{5}$ hypergeometric transformation; it later received another proof by one of these authors [29] via the WZ method [in fact, using the very same 'WZ certificate' as in [2] for (1.1)]. Notice that (1.2) is an example of supercongruence meaning that it holds modulo a power of $p$ greater than 1 .

Another entry on Van Hamme's 1997 list [28], tagged (H.2), is the congruence

$$
\sum_{k=0}^{(p-1) / 2} A_{k} \equiv \begin{cases}-\Gamma_{p}(1 / 4)^{4}\left(\bmod p^{2}\right) & \text { if } p \equiv 1(\bmod 4) \\ 0\left(\bmod p^{2}\right) & \text { if } p \equiv 3(\bmod 4)\end{cases}
$$

again for any $p>2$ prime, and $\Gamma_{p}(x)$ is the $p$-adic Gamma function. Van Hamme not only observed but also proved (1.3) in [28], and it was later generalized by Sun [23,24, Theorem 2.5], Guo and Zeng [12, Corollary 1.2], Long and Ramakrishna [17], Liu [15,16, Theorem 1.5] in different ways. For example, Long and Ramakrishna [17, Theorem 3] gave the following generalization of $(1.3)$ :

$$
\sum_{k=0}^{(p-1) / 2} A_{k} \equiv \begin{cases}-\Gamma_{p}(1 / 4)^{4}\left(\bmod p^{3}\right) & \text { if } p \equiv 1(\bmod 4) \\ -\frac{p^{2}}{16} \Gamma_{p}(1 / 4)^{4}\left(\bmod p^{3}\right) & \text { if } p \equiv 3(\bmod 4)\end{cases}
$$


Recently, these authors [14, Theorem 2] proved that, for any positive odd integer $n$, modulo $\Phi_{n}(q)^{2}$,

$$
\sum_{k=0}^{(n-1) / 2} \frac{\left(q ; q^{2}\right)_{k}^{2}\left(q^{2} ; q^{4}\right)_{k}}{\left(q^{2} ; q^{2}\right)_{k}^{2}\left(q^{4} ; q^{4}\right)_{k}} q^{2 k} \equiv \begin{cases}\frac{\left(q^{2} ; q^{4}\right)_{(n-1) / 4}^{2}}{\left(q^{4} ; q^{4}\right)_{(n-1) / 4}^{2}} q^{(n-1) / 2} & \text { if } n \equiv 1(\bmod 4) \\ 0 & \text { if } n \equiv 3(\bmod 4)\end{cases}
$$

Here and in what follows, $\Phi_{n}(q)$ denotes the $n$th cyclotomic polynomial; the $q$-shifted factorial is given by $(a ; q)_{0}=1$ and $(a ; q)_{n}=(1-a)(1-a q) \ldots$ $\left(1-a q^{n-1}\right)$ for $n \geqslant 1$ or $n=\infty$, while $[n]=[n]_{q}=1+q+\cdots+q^{n-1}$ stands for the $q$-integer. Van Hamme [27, Theorem 3] also proved that

$$
\left(\begin{array}{c}
-1 / 2 \\
(p-1) / 4
\end{array}\right) \equiv-\frac{\Gamma_{p}(1 / 4)^{2}}{\Gamma_{p}(1 / 2)}\left(\bmod p^{2}\right) ;
$$

in view of $\Gamma_{p}(1 / 2)^{2}=-1$ for $p \equiv 1(\bmod 4)$, by letting $q \rightarrow 1$ in $(1.5)$ for $n=p$ we immediately obtain (1.3).

One feature of (1.3) (not highlighted in [28]) is its connection with the coefficients

$$
a(p)= \begin{cases}2\left(a^{2}-b^{2}\right) & \text { if } p=a^{2}+b^{2}, a \text { odd }, \\ 0 & \text { if } p \equiv 3(\bmod 4),\end{cases}
$$

of CM modular form $q \prod_{j=1}^{\infty}\left(1-q^{4 j}\right)^{6}$ of weight 3 , namely, the congruence

$$
a(p) \equiv-\Gamma_{p}(1 / 4)^{4}\left(\bmod p^{2}\right) \quad \text { for primes } p \equiv 1(\bmod 4) .
$$

This served as a main motivation in [14] for not only establishing (1.5) but also speculating on possible $q$-deformation of modular forms.

For some other recent progress on $q$-analogues of supercongruences, the reader is referred to $[4,5,7-11,13,20,22,26,29]$. In particular, the authors [13] introduced and executed a new method of creative microscoping to prove (and reprove) many $q$-analogues of classical supercongruences and also raised some problems on $q$-congruences. Using this method, the first author [6] gave a refinement of (1.5) modulo $\Phi_{n}(q)^{3}$ for $n \equiv 3(\bmod 4)$, in other words, a $q$ analogue of $(1.4)$ for $p \equiv 3(\bmod 4)$.

A goal of this note is to present the following new $q$-analogue of Van Hamme's supercongruence (1.3).

Theorem 1.1. Let $n$ be a positive odd integer. Then

$$
\begin{aligned}
& \sum_{k=0}^{(n-1) / 2} \frac{\left(1+q^{4 k+1}\right)\left(q^{2} ; q^{4}\right)_{k}^{3}}{(1+q)\left(q^{4} ; q^{4}\right)_{k}^{3}} q^{k} \\
& \quad \equiv \frac{[n]_{q^{2}}\left(q^{3} ; q^{4}\right)_{(n-1) / 2}}{\left(q^{5} ; q^{4}\right)_{(n-1) / 2}} q^{(1-n) / 2} \begin{cases}\left(\bmod \Phi_{n}(q)^{2} \Phi_{n}(-q)^{3}\right) & \text { if } n \equiv 1(\bmod 4), \\
\left(\bmod \Phi_{n}(q)^{3} \Phi_{n}(-q)^{3}\right) & \text { if } n \equiv 3(\bmod 4)\end{cases}
\end{aligned}
$$


Note that $\Phi_{n}(q) \Phi_{n}(-q)=\Phi_{n}\left(q^{2}\right)$ for odd indices $n$.

The $n \equiv 3(\bmod 4)$ case of Theorem 1.1 confirms a conjecture of these authors [13, Conjecture 4.13], which states that, for $n \equiv 3(\bmod 4)$,

$$
\sum_{k=0}^{(n-1) / 2} \frac{\left(1+q^{4 k+1}\right)\left(q^{2} ; q^{4}\right)_{k}^{3}}{(1+q)\left(q^{4} ; q^{4}\right)_{k}^{3}} q^{k} \equiv 0\left(\bmod \Phi_{n}(q)^{2} \Phi_{n}(-q)\right) .
$$

It is not difficult to verify that

$$
\frac{(3 / 4)_{(p-1) / 2}}{(5 / 4)_{(p-1) / 2}} \equiv-\frac{p}{16} \Gamma_{p}(1 / 4)^{4}\left(\bmod p^{2}\right)
$$

for $p \equiv 3(\bmod 4)$, where $(a)_{n}=a(a+1) \ldots(a+n-1)$ denotes the rising factorial (also known as Pochhammer's symbol). Therefore, the $q$-congruence (1.7) reduces to $(1.4)$ for $p \equiv 3(\bmod 4)$ when $n=p$ and $q \rightarrow 1$, and it reduces to $(1.3)$ for $p \equiv 1(\bmod 4)$ when $n=p$ and $q \rightarrow 1$. Moreover, letting $n=p$ and $q \rightarrow-1$ in (1.7), we immediately get (1.2). Thus, Theorem 1.1 presents a common $q$-analogue of supercongruences (1.2) and (1.3). We point out that other different $q$-analogues of $(1.2)$ have been given in $[7,8]$.

Recently, Mao and Pan [18] (see also Sun [25, Theorem 1.3]) proved that, if $p \equiv 1(\bmod 4)$ is a prime, then

$$
\sum_{k=0}^{(p+1) / 2} \frac{(-1 / 2)_{k}^{3}}{k !^{3}} \equiv 0\left(\bmod p^{2}\right)
$$

In this note, we prove the following $q$-analogue of (1.8).

Theorem 1.2. Let $n>1$ be an odd integer. Then

$$
\begin{aligned}
& \sum_{k=0}^{(n+1) / 2} \frac{\left(1+q^{4 k-1}\right)\left(q^{-2} ; q^{4}\right)_{k}^{3}}{(1+q)\left(q^{4} ; q^{4}\right)_{k}^{3}} q^{7 k} \\
& \quad \equiv \frac{[n]_{q^{2}}\left(q ; q^{4}\right)_{(n-1) / 2}}{\left(q^{7} ; q^{4}\right)_{(n-1) / 2}} q^{(n-3) / 2} \begin{cases}\left(\bmod \Phi_{n}(q)^{3} \Phi_{n}(-q)^{3}\right) & \text { if } n \equiv 1(\bmod 4), \\
\left(\bmod \Phi_{n}(q)^{2} \Phi_{n}(-q)^{3}\right) & \text { if } n \equiv 3(\bmod 4) .\end{cases}
\end{aligned}
$$

The $n \equiv 1(\bmod 4)$ case of Theorem 1.2 also confirms a conjecture of the first author and Schlosser [11, Conjecture 10.2].

For $n$ prime, letting $q \rightarrow 1$ in Theorem 1.2 we obtain the following generalization of (1.8).

Corollary 1.3. Let $p$ be an odd prime. Then

$$
\sum_{k=0}^{(p+1) / 2} \frac{(-1 / 2)_{k}^{3}}{k !^{3}} \equiv p \frac{(1 / 4)_{(p-1) / 2}}{(7 / 4)_{(p-1) / 2}} \begin{cases}\left(\bmod p^{3}\right) & \text { if } p \equiv 1(\bmod 4), \\ \left(\bmod p^{2}\right) & \text { if } p \equiv 3(\bmod 4) .\end{cases}
$$

On the other hand, for $n$ prime and $q \rightarrow-1$ in Theorem 1.2, we are led to the following result:

$$
\sum_{k=0}^{(p+1) / 2}(-1)^{k}(4 k-1) \frac{(-1 / 2)_{k}^{3}}{k !^{3}} \equiv p(-1)^{(p+1) / 2}\left(\bmod p^{3}\right) .
$$


It should be mentioned that a different $q$-analogue of (1.9) was given in [13, Theorem 4.9] with $r=-1, d=2$ and $a=1$ (see also [11, Section 5]).

Moreover, for the summation formula

$$
\sum_{k=0}^{\infty} \frac{\left(-\frac{1}{2}\right)_{k}^{3}}{k !^{3}}=12 \frac{\Gamma(3 / 4)^{4}}{\pi^{3}}
$$

we have the following $q$-analogue.

Theorem 1.4. We have

$$
\sum_{k=0}^{\infty} \frac{\left(1+q^{4 k-1}\right)\left(q^{-2} ; q^{4}\right)_{k}^{3}}{\left(1+q^{-1}\right)\left(q^{4} ; q^{4}\right)_{k}^{3}} q^{7 k}=\frac{\left(q^{2} ; q^{4}\right)_{\infty}\left(q^{5} ; q^{4}\right)_{\infty}^{2}\left(q^{6} ; q^{4}\right)_{\infty}}{\left(q^{3} ; q^{4}\right)_{\infty}\left(q^{4} ; q^{4}\right)_{\infty}^{2}\left(q^{7} ; q^{4}\right)_{\infty}}
$$

Both Theorems 1.1 and 1.2 are particular cases of a more general result, which we state and prove in the next section, while Theorem 1.4 follows from a classical $q$-identity.

\section{A Family of $q$-Congruences from the $q$-Dixon Sum}

In this section we establish the following family of one-parameter $q$-congruences.

Theorem 2.1. Let $n \geqslant 1$ be an odd integer and $\ell$ an integer with $0 \leqslant \ell \leqslant$ $(n-1) / 2$. Then

$$
\begin{aligned}
& \sum_{k=0}^{n-1} \frac{\left(1+q^{4 k-2 \ell+1}\right)\left(q^{2-4 \ell} ; q^{4}\right)_{k}^{3}}{\left(1+q^{1-2 \ell}\right)\left(q^{4} ; q^{4}\right)_{k}^{3}} q^{(6 \ell+1) k} \\
& \equiv \frac{\left(1-q^{2 n}\right)\left(q^{3-6 \ell} ; q^{4}\right)_{(n-1) / 2+\ell}}{\left(1-q^{2-4 \ell}\right)\left(q^{5-2 \ell} ; q^{4}\right)_{(n-1) / 2+\ell}} q^{(2 \ell-1)((n-1) / 2+\ell)}\left\{\begin{array}{c}
\left(\bmod \Phi_{n}(q)^{2} \Phi_{n}(-q)^{3}\right) \\
\text { if } n+2 \ell \equiv 1(\bmod 4) \\
\left(\bmod \Phi_{n}(q)^{3} \Phi_{n}(-q)^{3}\right) \\
\text { if } n+2 \ell \equiv 3(\bmod 4)
\end{array}\right.
\end{aligned}
$$

Note that the $q$-congruence (2.1) remains true when the sum is over $k$ from 0 to $(n-1) / 2+\ell$, since $\left(q^{2-4 \ell} ; q^{4}\right)_{k} /\left(q^{4} ; q^{4}\right)_{k} \equiv 0\left(\bmod \Phi_{n}\left(q^{2}\right)\right)$ for $(n-1) / 2+\ell<k \leqslant n-1$. Furthermore, when $\ell=0$ and $\ell=1$ (hence $n \geq 3$ ) the theorem reduces to Theorems 1.1 and 1.2 , respectively.

The following easily proved $q$-congruence (see [11, Lemma 3.1]) is necessary in our derivation of Theorem 2.1.

Lemma 2.2. Let $n$ be a positive odd integer. Then, for $0 \leqslant k \leqslant(n-1) / 2$, we have

$$
\frac{\left(a q ; q^{2}\right)_{(n-1) / 2-k}}{\left(q^{2} / a ; q^{2}\right)_{(n-1) / 2-k}} \equiv(-a)^{(n-1) / 2-2 k} \frac{\left(a q ; q^{2}\right)_{k}}{\left(q^{2} / a ; q^{2}\right)_{k}} q^{(n-1)^{2} / 4+k}\left(\bmod \Phi_{n}(q)\right) .
$$

Like the proofs given in [13], we start with the following generalization of (1.7) with an extra parameter $a$. 
Theorem 2.3. Let $n>1$ be an odd integer and $0 \leqslant \ell \leqslant(n-1) / 2$. Then

$$
\begin{aligned}
& \sum_{k=0}^{n-1} \frac{\left(1+q^{4 k-2 \ell+1}\right)\left(a q^{2-4 \ell} ; q^{4}\right)_{k}\left(q^{2-4 \ell} / a ; q^{4}\right)_{k}\left(q^{2-4 \ell} ; q^{4}\right)_{k}}{\left(1+q^{1-2 \ell}\right)\left(a q^{4} ; q^{4}\right)_{k}\left(q^{4} / a ; q^{4}\right)_{k}\left(q^{4} ; q^{4}\right)_{k}} q^{(6 \ell+1) k} \\
& \equiv \frac{\left(1-q^{2 n}\right)\left(q^{3-6 \ell} ; q^{4}\right)_{(n-1) / 2+\ell}}{\left(1-q^{2-4 \ell}\right)\left(q^{5-2 \ell} ; q^{4}\right)_{(n-1) / 2+\ell}} \\
& \times q^{(2 \ell-1)((n-1) / 2+\ell)}\left\{\begin{array}{c}
\left(\bmod \Phi_{n}(-q)\left(1-a q^{2 n}\right)\left(a-q^{2 n}\right)\right) \\
\text { if } n+2 \ell \equiv 1(\bmod 4), \\
\left(\bmod \Phi_{n}\left(q^{2}\right)\left(1-a q^{2 n}\right)\left(a-q^{2 n}\right)\right) \\
\text { if } n+2 \ell \equiv 3(\bmod 4) .
\end{array}\right.
\end{aligned}
$$

Proof. Performing the parameter substitutions $q \mapsto q^{4}, a \mapsto q^{2-4 \ell}, b \mapsto b q^{2-4 \ell}$ and $c \mapsto c q^{2-4 \ell}$ in the $q$-Dixon sum [3, Appendix (II.13)], we obtain

$$
\begin{gathered}
\left.\left.\sum_{k=0}^{\infty} \frac{\left(1+q^{4 k-2 \ell+1}\right)\left(q^{2-4 \ell} ; q^{4}\right)_{k}\left(b q^{2-4 \ell} ; q^{4}\right)_{k}\left(c q^{2-4 \ell} ; q^{4}\right)_{k}}{\left(1+q^{1-2 \ell}\right)\left(q^{4} / b ; q^{4}\right)_{k}\left(q^{4} / c ; q^{4}\right)_{k}\left(q^{4} ; q^{4}\right)_{k}}\right)^{6 \ell+1}\right)^{k} \\
\quad=\frac{\left(q^{6-4 \ell} ; q^{4}\right)_{\infty}\left(q^{2 \ell+3} / b ; q^{4}\right)_{\infty}\left(q^{2 \ell+3} / c ; q^{4}\right)_{\infty}\left(q^{4 \ell+2} / b c ; q^{4}\right)_{\infty}}{\left(q^{4} / b ; q^{4}\right)_{\infty}\left(q^{4} / c ; q^{4}\right)_{\infty}\left(q^{5-2 \ell} ; q^{4}\right)_{\infty}\left(q^{6 \ell+1} / b c ; q^{4}\right)_{\infty}}
\end{gathered}
$$

Since $n$ is odd, putting $b=q^{-2 n}$ and $c=q^{2 n}$ in (2.3) we see that the left-hand side terminates and is equal to

$$
\begin{gathered}
\sum_{k=0}^{(n-1) / 2+\ell} \frac{\left(1+q^{4 k-2 \ell+1}\right)\left(q^{2-4 \ell-2 n} ; q^{4}\right)_{k}\left(q^{2-4 \ell+2 n} ; q^{4}\right)_{k}\left(q^{2-4 \ell} ; q^{4}\right)_{k}}{\left(1+q^{1-2 \ell}\right)\left(q^{4-2 n} ; q^{4}\right)_{k}\left(q^{4+2 n} ; q^{4}\right)_{k}\left(q^{4} ; q^{4}\right)_{k}} q^{(6 \ell+1) k} \\
=\sum_{k=0}^{n-1} \frac{\left(1+q^{4 k-2 \ell+1}\right)\left(q^{2-4 \ell-2 n} ; q^{4}\right)_{k}\left(q^{2-4 \ell+2 n} ; q^{4}\right)_{k}\left(q^{2-4 \ell} ; q^{4}\right)_{k}}{\left(1+q^{1-2 \ell}\right)\left(q^{4-2 n} ; q^{4}\right)_{k}\left(q^{4+2 n} ; q^{4}\right)_{k}\left(q^{4} ; q^{4}\right)_{k}} q^{(6 \ell+1) k}
\end{gathered}
$$

while the right-hand side becomes

$$
\begin{aligned}
& \frac{\left(q^{2 \ell-2 n+3} ; q^{4}\right)_{(n-1) / 2+\ell}\left(q^{6-4 \ell} ; q^{4}\right)_{(n-1) / 2+\ell}}{\left(q^{4-2 n} ; q^{4}\right)_{(n-1) / 2+\ell}\left(q^{5-2 \ell} ; q^{4}\right)_{(n-1) / 2+\ell}} \\
& \quad=\frac{\left(1-q^{2 n}\right)\left(q^{3-6 \ell} ; q^{4}\right)_{(n-1) / 2+\ell}}{\left(1-q^{2-4 \ell}\right)\left(q^{5-2 \ell} ; q^{4}\right)_{(n-1) / 2+\ell}} q^{(2 \ell-1)((n-1) / 2+\ell)} .
\end{aligned}
$$

This proves that the $q$-congruence (2.2) holds modulo $1-a q^{2 n}$ or $a-q^{2 n}$.

On the other hand, by Lemma 2.2 , for $0 \leqslant k \leqslant(n-1) / 2+\ell$, modulo $\Phi_{n}(q)$ we have 


$$
\begin{aligned}
& \frac{\left(a q^{1-2 \ell} ; q^{2}\right)_{(n-1) / 2+\ell-k}}{\left(q^{2} / a ; q^{2}\right)_{(n-1) / 2+\ell-k}} \\
& \quad=\frac{\left(a q^{1-2 \ell} ; q^{2}\right)_{\ell}\left(a q ; q^{2}\right)_{(n-1) / 2-k}}{\left(q^{n+1-2 k} / a ; q^{2}\right)_{\ell}\left(q^{2} / a ; q^{2}\right)_{(n-1) / 2-k}} \\
& \quad \equiv(-a)^{(n-1) / 2-2 k} \frac{\left(a q^{1-2 \ell} ; q^{2}\right)_{\ell}\left(a q ; q^{2}\right)_{k}}{\left(q^{n+1-2 k} / a ; q^{2}\right)_{\ell}\left(q^{2} / a ; q^{2}\right)_{k}} q^{(n-1)^{2} / 4+k} \\
& \quad=(-a)^{(n-1) / 2-2 k} \frac{\left(a q^{1-2 \ell} ; q^{2}\right)_{k}\left(a q^{2 k-2 \ell+1} ; q^{2}\right)_{\ell}}{\left(q^{n+1-2 k} / a ; q^{2}\right)_{\ell}\left(q^{2} / a ; q^{2}\right)_{k}} q^{(n-1)^{2} / 4+k} \\
& \quad \equiv(-a)^{(n-1) / 2+\ell-2 k} \frac{\left(a q^{1-2 \ell} ; q^{2}\right)_{k}}{\left(q^{2} / a ; q^{2}\right)_{k}} q^{(n-1)^{2} / 4+k+(2 k-\ell) \ell},
\end{aligned}
$$

where we used $q^{n} \equiv 1\left(\bmod \Phi_{n}(q)\right)$ in the last step. Using the above $q$ congruence we can easily check that, for odd $n>1$ and $0 \leqslant k \leqslant(n-1) / 2+\ell$, sum of the $k$ th and $((n-1) / 2+\ell-k)$ th summands on the left-hand side of (2.2) is congruent to 0 modulo $\Phi_{n}(-q)$ (or modulo $\Phi_{n}\left(q^{2}\right)$ if $n \equiv 3-2 \ell$ $(\bmod 4))$. It follows that

$$
\begin{aligned}
& \sum_{k=0}^{(n-1) / 2+\ell} \frac{\left(1+q^{4 k-2 \ell+1}\right)\left(a q^{2-4 \ell} ; q^{4}\right)_{k}\left(q^{2-4 \ell} / a ; q^{4}\right)_{k}\left(q^{2-4 \ell} ; q^{4}\right)_{k}}{\left(1+q^{1-2 \ell}\right)\left(a q^{4} ; q^{4}\right)_{k}\left(q^{4} / a ; q^{4}\right)_{k}\left(q^{4} ; q^{4}\right)_{k}} q^{(6 \ell+1) k} \\
& \equiv 0 \begin{cases}\left(\bmod \Phi_{n}(-q)\right) & \text { if } n+2 \ell \equiv 1(\bmod 4), \\
\left(\bmod \Phi_{n}\left(q^{2}\right)\right) & \text { if } n+2 \ell \equiv 3(\bmod 4) .\end{cases}
\end{aligned}
$$

Clearly, the right-hand side of $(2.1)$ is congruent to 0 modulo $\Phi_{n}(-q)$ if $n+$ $2 \ell \equiv 1(\bmod 4)$ and modulo $\Phi_{n}\left(q^{2}\right)$ if $n+2 \ell \equiv 3(\bmod 4)$. Therefore, the $q$-congruence $(2.2)$ holds modulo $\Phi_{n}(-q)$ if $n+2 \ell \equiv 1(\bmod 4)$ and modulo $\Phi_{n}\left(q^{2}\right)$ if $n+2 \ell \equiv 3(\bmod 4)$. Since the polynomials $1-a q^{2 n}, a-q^{2 n}$ and $\Phi_{n}(-q)$ (or $\Phi_{n}\left(q^{2}\right)$ ) are pairwise coprime, we complete the proof of $(2.2)$.

Proof of Theorem 2.1. We assume that $n>1$, since the $n=1$ case (making $\ell=0$ only possible) is trivial. The limits of the denominators on both sides of $(2.2)$ as $a \rightarrow 1$ are relatively prime to $\Phi_{n}\left(q^{2}\right)$, since $k$ is in the range $0 \leqslant k \leqslant(n-1) / 2+\ell$. On the other hand, the limit of $\left(1-a q^{2 n}\right)\left(a-q^{2 n}\right)$ as $a \rightarrow 1$ contains the factor $\Phi_{n}\left(q^{2}\right)^{2}$.

Proof of Theorem 1.4. Take $b=c=\ell=1$ in Eq. (2.3).

\section{Discussion}

The method of creative microscoping used in our proofs indicates the origin of $q$-congruences from infinite $q$-hypergeometric identities; for example, the $q$-congruence (1.7) corresponds to the identity

$$
\sum_{k=0}^{\infty} \frac{\left(1+q^{4 k+1}\right)\left(q^{2} ; q^{4}\right)_{k}^{3}}{(1+q)\left(q^{4} ; q^{4}\right)_{k}^{3}} q^{k}=\frac{\left(q^{2} ; q^{4}\right)_{\infty}^{2}\left(q^{3} ; q^{4}\right)_{\infty}^{2}}{(1+q)\left(q ; q^{4}\right)_{\infty}^{2}\left(q^{4} ; q^{4}\right)_{\infty}^{2}}
$$


which is just a particular instance of (2.3). Note that the limiting cases as $q \rightarrow-1$ and $q \rightarrow 1$ of (3.1) give the formulas (1.1) and

$$
\sum_{k=0}^{\infty} \frac{\left(\frac{1}{2}\right)_{k}^{3}}{k !^{3}}=\frac{\Gamma(1 / 4)^{4}}{4 \pi^{3}}=\frac{8 L(f, 1)}{\pi}=\frac{16 L(f, 2)}{\pi^{2}}
$$

where

$$
f(\tau)=q \prod_{j=1}^{\infty}\left(1-q^{4 j}\right)^{6}=\sum_{n=1}^{\infty} a(n) q^{n}, \quad \text { with } q=\exp (2 \pi i \tau),
$$

is the CM modular form from the introduction and $L(f, s)$ denotes its $L$-function. This means that the $q$-identity (3.1) presents a common $q$-extension of evaluations (1.1) and (3.2) - the fact that makes it less surprising that the $q$-congruence (1.7) simultaneously extends (1.2) and (1.3).

The intermediate use of parametric $q$-hypergeometric identities in our proof of Theorem 2.1 based on the $q$-Dixon sum suggests that different $q$ congruences underlying (3.1) are possible. This is indeed the case when we analyze the formula (3.1) as the $a=1$ specialization of

$$
\begin{gathered}
\sum_{k=0}^{\infty} \frac{\left(1+q^{4 k+1}\right)\left(a q ; q^{2}\right)_{k}\left(q / a ; q^{2}\right)_{k}\left(-q ; q^{2}\right)_{k}^{2}\left(q^{2} ; q^{4}\right)_{k}}{(1+q)\left(q^{2} ; q^{2}\right)_{k}^{2}\left(-a q^{2} ; q^{2}\right)_{k}\left(-q^{2} / a ; q^{2}\right)_{k}\left(q^{4} ; q^{4}\right)_{k}} q^{k} \\
\quad=\frac{\left(-q ; q^{2}\right)_{\infty}^{2}\left(a q^{3} ; q^{4}\right)_{\infty}^{2}\left(q^{3} / a ; q^{4}\right)_{\infty}^{2}}{(1+q)\left(-a q^{2} ; q^{2}\right)_{\infty}\left(-q^{2} / a ; q^{2}\right)_{\infty}\left(q^{2} ; q^{2}\right)_{\infty}^{2}}
\end{gathered}
$$

which originates from a $q$-analogue of Watson's ${ }_{3} F_{2}$ sum [3, Appendix (II.16)]. When we choose $a=q^{n}$ (or $a=q^{-n}$ ) in (3.3), for $n>1$ odd, we get the sum terminating after $(n-1) / 2$ terms on the left-hand side of $(3.3)$, while the right-hand side vanishes if $n$ is of the form $4 m+3$ and it becomes equal to

$$
\frac{\left(-q ; q^{2}\right)_{\infty}^{2}\left(q^{4 m+4} ; q^{4}\right)_{\infty}^{2}\left(q^{2-4 m} ; q^{4}\right)_{\infty}^{2}}{(1+q)\left(-q^{4 m+3} ; q^{2}\right)_{\infty}\left(-q^{1-4 m} ; q^{2}\right)_{\infty}\left(q^{2}, q^{4} ; q^{4}\right)_{\infty}^{2}}=[4 m+1] \frac{\left(q^{2} ; q^{4}\right)_{m}^{2}}{\left(q^{4} ; q^{4}\right)_{m}^{2}}
$$

if $n=4 m+1$. This means that modulo $\left(a-q^{n}\right)\left(1-a q^{n}\right)$ we have

$$
\begin{aligned}
& \sum_{k=0}^{N} \frac{\left(1+q^{4 k+1}\right)\left(a q ; q^{2}\right)_{k}\left(q / a ; q^{2}\right)_{k}\left(-q ; q^{2}\right)_{k}^{2}\left(q^{2} ; q^{4}\right)_{k}}{(1+q)\left(q^{2} ; q^{2}\right)_{k}^{2}\left(-a q^{2} ; q^{2}\right)_{k}\left(-q^{2} / a ; q^{2}\right)_{k}\left(q^{4} ; q^{4}\right)_{k}} q^{k} \\
& \quad \equiv \begin{cases}{[4 m+1] \frac{\left(q^{2} ; q^{4}\right)_{m}^{2}}{\left(q^{4} ; q^{4}\right)_{m}^{2}}} & \text { if } n=4 m+1, \\
0 & \text { if } n \equiv 3(\bmod 4),\end{cases}
\end{aligned}
$$

for any $N \geq(n-1) / 2$. The limiting $a \rightarrow 1$ case of the congruences can be shown to be

$$
\sum_{k=0}^{(n-1) / 2} \frac{\left(1+q^{4 k+1}\right)\left(q^{2} ; q^{4}\right)_{k}^{3}}{(1+q)\left(q^{4} ; q^{4}\right)_{k}^{3}} q^{k} \equiv \begin{cases}{[4 m+1] \frac{\left(q^{2} ; q^{4}\right)_{m}^{2}}{\left(q^{4} ; q^{4}\right)_{m}^{2}}} & \text { if } n=4 m+1 \\ 0 & \text { if } n \equiv 3(\bmod 4)\end{cases}
$$


modulo $\Phi_{n}(q)^{2} \Phi_{n}(-q)$. This is quite similar in spirit to (1.5), though still far from constructing $q$-analogues for the coefficients $a(p)$ in (1.6) of the modular form $f(\tau)$. The latter means that a hunt for $q$-rational functions, which equal the left-hand side of (1.5) or (3.4) modulo $\Phi_{n}(q)^{2}$ and specialize to $a(n)$ as $q \rightarrow 1$ (at least for $n$ prime), is still on its way. Such $q$-rational functions are also expected to be self-reciprocal, that is, invariant under the involution $q \mapsto 1 / q$, as all the left- and right-hand sides in (1.5), (1.7), (3.4) and also (2.1) are.

Open Access. This article is licensed under a Creative Commons Attribution 4.0 International License, which permits use, sharing, adaptation, distribution and reproduction in any medium or format, as long as you give appropriate credit to the original author(s) and the source, provide a link to the Creative Commons licence, and indicate if changes were made. The images or other third party material in this article are included in the article's Creative Commons licence, unless indicated otherwise in a credit line to the material. If material is not included in the article's Creative Commons licence and your intended use is not permitted by statutory regulation or exceeds the permitted use, you will need to obtain permission directly from the copyright holder. To view a copy of this licence, visit http://creativecommons. org/licenses/by/4.0/.

\section{References}

[1] Bauer, G.: Von den coefficienten der Reihen von Kugelfunctionen einer variabeln. J. Reine Angew. Math. 56, 101-121 (1859)

[2] Ekhad, S.B., Zeilberger, D., WZ, A.: Proof of Ramanujan's formula for $\pi$. In: Rassias, J.M. (ed.) Geometry, Analysis, and Mechanics, p. 107. World Scientific, Singapore (1994)

[3] Gasper, G., Rahman, M.: Basic Hypergeometric Series (Encyclopedia of Mathematics and Its Applications 96), 2nd edn. Cambridge University Press, Cambridge (2004)

[4] Gorodetsky, O.: q-Congruences, with applications to supercongruences and the cyclic sieving phenomenon. Int. J. Number Theory 15, 1919-1968 (2019)

[5] Gu, C.-Y., Guo, V.J.W.: q-Analogues of two supercongruences of Z.-W. Sun. Czechoslovak Math. J. (2020). https://doi.org/10.21136/CMJ.2020.0516-18

[6] Guo, V.J.W.: A further $q$-analogue of Van Hamme's (H.2) supercongruence for primes $p \equiv 3(\bmod 4)$, preprint $(2019)$

[7] Guo, V.J.W.: A $q$-analogue of a Ramanujan-type supercongruence involving central binomial coefficients. J. Math. Anal. Appl. 458, 590-600 (2018)

[8] Guo, V.J.W.: Common $q$-analogues of some different supercongruences. Results Math. 74, 131 (2019)

[9] Guo, V.J.W.: q-Analogues of two "divergent" Ramanujan-type supercongruences. Ramanujan J. (2020). https://doi.org/10.1007/s11139-019-00161-0

[10] Guo, V.J.W., Schlosser, M.J.: Some new $q$-congruences for truncated basic hypergeometric series: even powers. Results Math. 75, 1 (2020) 
[11] Guo, V.J.W., Schlosser, M.J.: Some $q$-supercongruences from transformation formulas for basic hypergeometric series. Constr. Approx. (to appear)

[12] Guo, V.J.W., Zeng, J.: Some $q$-supercongruences for truncated basic hypergeometric series. Acta Arith. 171, 309-326 (2015)

[13] Guo, V.J.W., Zudilin, W.: A q-microscope for supercongruences. Adv. Math. 346, 329-358 (2019)

[14] Guo, V.J.W., Zudilin, W.: On a $q$-deformation of modular forms. J. Math. Anal. Appl. 475, 1636-646 (2019)

[15] Liu, J.-C.: Some supercongruences on truncated ${ }_{3} F_{2}$ hypergeometric series. J. Differ. Equ. Appl. 24, 438-451 (2018)

[16] Liu, J.-C.: On Van Hamme's (A.2) and (H.2) supercongruences. J. Math. Anal. Appl. 471, 613-622 (2019)

[17] Long, L., Ramakrishna, R.: Some supercongruences occurring in truncated hypergeometric series. Adv. Math. 290, 773-808 (2016)

[18] Mao, G.-S., Pan, H.: On the divisibility of some truncated hypergeometric series. Acta Arith. (to appear)

[19] Mortenson, E.: A p-adic supercongruence conjecture of van Hamme. Proc. Am. Math. Soc. 136, 4321-4328 (2008)

[20] Ni, H.-X., Pan, H.: On a conjectured $q$-congruence of Guo and Zeng. Int. J. Number Theory 14, 1699-1707 (2018)

[21] Ramanujan, S.: Modular equations and approximations to $\pi$. Quart. J. Math. Oxf. Ser. 2(45), 350-372 (1914)

[22] Straub, A.: Supercongruences for polynomial analogs of the Apéry numbers. Proc. Am. Math. Soc. 147, 1023-1036 (2019)

[23] Sun, Z.-H.: Congruences concerning Legendre polynomials II. J. Number Theory 133, 1950-1976 (2013)

[24] Sun, Z.-H.: Generalized Legendre polynomials and related supercongruences. J. Number Theory 143, 293-319 (2014)

[25] Sun, Z.-W.: On sums of Apéry polynomials and related congruences. J. Number Theory 132, 2673-2690 (2012)

[26] Tauraso, R.: q-Analogs of some congruences involving Catalan numbers. Adv. Appl. Math. 48, 603-614 (2009)

[27] Van Hamme, L.: Proof of a conjecture of Beukers on Apéry numbers. In: Proceedings of the Conference on p-Adic Analysis (Houthalen, 1987), pp. 189-195. Vrije Univ. Brussel, Brussels (1986)

[28] Van Hamme, L.: Some conjectures concerning partial sums of generalized hypergeometric series. In: $p$-Adic Functional Analysis (Nijmegen, 1996), Lecture Notes in Pure and Applied Mathematics, vol. 192, pp. 223-236. Dekker, New York (1997)

[29] Zudilin, W.: Ramanujan-type supercongruences. J. Number Theory 129, 1848$1857(2009)$ 
Victor J. W. Guo

School of Mathematics and Statistics

Huaiyin Normal University

Huai'an 223300

Jiangsu

People's Republic of China

e-mail: jwguo@hytc.edu.cn

Wadim Zudilin

Department of Mathematics, IMAPP

Radboud University

PO Box 9010

6500 GL Nijmegen

The Netherlands

e-mail: w.zudilin@math.ru.nl

Received: October 30, 2019.

Accepted: February 3, 2020.

Publisher's Note Springer Nature remains neutral with regard to jurisdictional claims in published maps and institutional affiliations. 